\title{
Small Intestinal Bacterial Overgrowth in Children with Functional Dyspepsia and Duodenal Ulcer Disease, Associated with Helicobacter Pylori Infection
}

\author{
Nalyotov $\mathrm{AV}^{* 1}$, Prokhorov $\mathrm{EV}^{2}$ and Masyuta $\mathrm{DI}^{1}$ \\ ${ }^{1}$ Department of Pediatrics and Childhood Infections, Donetsk National Medical University of Maxim Gorky, \\ Donetsk, Ukraine \\ ${ }^{2}$ Department of Pediatrics, Donetsk National Medical University of Maxim Gorky, Donetsk, Ukraine
}

*Corresponding author: Nalyotov AV, Department of Pediatrics and Childhood Infections, Donetsk National Medical University of Maxim Gorky, Illicha Avenue, 16, Donetsk 283003, Ukraine; E-mail: nalyotov-a@mail.ru

Citation: Nalyotov AV, Prokhorov EV, Masyuta DI (2016) Small Intestinal Bacterial Overgrowth in Children with Functional Dyspepsia and Duodenal Ulcer Disease, Associated with Helicobacter Pylori Infection. J Gastroenterol Compl 1(1): 105

Received Date: July 01, 2016 Accepted Date: November 09, 2016 Published Date: November 11, 2016

\begin{abstract}
Summary: Recent studies suggest a potential relationship between helicobacter pylori (HP) infection and small intestinal bacterial overgrowth (SIBO). The state of the digestive tract microflora is not often taken in account in the treatment of children with chronic gastroduodenal pathology, associated with HP. The purpose of this study was to determine the frequency of SIBO in children with duodenal ulcer disease (DUD) and functional dyspepsia, associated with HP.

Methods: 60 patients with DUD and 50 children with functional dyspepsia, aged 12 to 17 years, were included in the study. Chronic gastroduodenal pathology was associated with HP infection in all patients. 30 healthy children were the control group. HP infection was detected in patients by the two ways: a rapid urease test with biopsy material and a urea breath test. Diagnosis of SIBO was based on a non-invasive hydrogen breath test with a load of lactulose.

Results: SIBO was detected in $57(95.0 \pm 2.8 \%)$ children with DUD, associated with HP. In $37(74.0 \pm 6.2 \%)$ patients with functional dyspepsia, associated with HP, SIBO was determined. In the control group SIBO was diagnosed only in $4(13.3 \pm 6.2 \%)$ children. The differences in frequency of SIBO between patients with DUD, children with functional dyspepsia, associated with HP, and children of the control group were statistically significant $[\mathrm{p}<0.05]$.

Conclusion: This study demonstrated the high prevalence of SIBO in patients with DUD and functional dyspepsia, associated with HP. It can lead to lower quality of eradication of HP and increase the frequency of side effects of this therapy.

Keywords: Children; Duodenal ulcer Disease; Functional Dyspepsia; Small Intestinal Bacterial Overgrowth; Helicobacter Pylori

List of abbreviations: DUD: Duodenal Ulcer Disease; SIBO: Small Intestinal Bacterial Overgrowth; HP: Helicobacter Pylori
\end{abstract}

\section{Introduction}

Helicobacter pylori (HP) infection is present in more than the half of the world population. Most individuals are infected during early childhood; in developing countries, $50 \%$ of children are infected by the age of 5 years [1,2]. Studies suggest the main etiological role of HP infection in development of chronic gastroduodenal pathology and the formation of its most severe forms in patients of different age groups. However, not all infected people exhibit diseases associated with this bacterium. In addition, pathogenic mechanisms of duodenal ulcer disease (DUD) in childhood remain not fully understood.

Human intestinal microbiota creates complex polymicrobial ecology. This is characterized by its high population density, wide diversity and complexity of interaction. The duodenum and the proximal jejunum normally contain small numbers of bacteria $[3,4]$. Small intestinal bacterial overgrowth (SIBO) is a very heterogeneous syndrome characterized by an increased number and/ or abnormal type of bacteria in the small intestine. Most authors consider diagnosis of SIBO to be the finding of $\geq 10^{5}$ bacteria [i.e. Colonyforming units] per ml of proximal jejunal aspiration. The SIBO is characterised by a large variety of clinical manifestations $[5,6]$. Symptoms of SIBO are directly associated with bacterial metabolism, intestinal mucosa damage that leads to impaired absorption of fat, proteins, carbohydrates, and fat-soluble vitamins [4-6]. The most commonly reported symptoms of SIBO include diarrhea, abdominal pain, and flatulence. The course of SIBO is also considered to be completely asymptomatic [5]. 
The standardization of the methods used in the diagnosis of SIBO is still under discussion. Some authors consider that the only method of diagnosing SIBO, regarded as the "gold standard", is microbiological examination of the content sampled from the proximal part of the small intestine. It is a direct method classified as an invasive one, as well as quite expensive $[4,6]$. There are still no established procedures regarding the use of samples and microbiological techniques. Thus, commonly used tests in the diagnosis of SIBO, especially in the pediatric population, are indirect methods, which include a hydrogen breath tests. The hydrogen breath test with a load of lactulose has been proposed to diagnose SIBO [7]. It is based on following breath hydrogen levels after the administration of a lactulose to a patient with suspected SIBO. The test is based on the interaction between the administered carbohydrate and the intestinal bacteria. The resulting fermentation produces hydrogen. A positive breath test is based on a breath hydrogen rise prior to the expected arrival time in the highly microbial cecum [8].

Recent studies suggest a potential relationship between chronic gastroduodenal pathology, associated with HP infection and SIBO [9]. HP has been implicated in allowing the passage of live pathogens beyond the stomach through impairing of stomach acid secretion and development of gastric mucosa inflammation. SIBO disturbs digestion and absorption in the alimentary tract, which seems to cause inflammation. Impaired barrier function results in changing luminal contents (including both harmless and potentially pathogenic bacteria) crossing the gut wall itself and activating the immune system. Long term SIBO may lead to disruption of the immune system and maintain inflammation throughout the digestive tract. The state of the digestive tract microflora is not often taken in account in the treatment of patients with DUD and functional dyspepsia, associated with HP. This fact can be considered as one of the causes of the poor effect of HP eradication therapy.

The purpose of this study was to determine the frequency of SIBO in children with DUD and functional dyspepsia, associated with HP.

\section{Materials and Methods}

The study was a monocentric trial. It was conducted at the medical center «Gastro line» Donetsk, Ukraine. All patients were enrolled in the study from January 2013 to January 2014. 60 patients with DUD were the group I (M/F 35/25, the median age was 14.5 years, the age range was from 12 to 17$)$, and 50 children with functional dyspepsia were the group II (M/F 35/23, the median age was 14.1 years, the age range was from 12 to 17). DUD was diagnosed by endoscopy. Ulcerative defects of the mucosa were detected during endoscopy in all patients of group I. The diagnosis of functional dyspepsia was based on Rome III criteria. Patients of group II complained of persistent or recurrent pain or discomfort centered in the upper abdomen. However they did not have any evidences of structural diseases (including at upper endoscopy) that is likely to explain the symptoms. Chronic gastroduodenal pathology was associated with HP infection in all patients, which was diagnosed for the first time. The control group consisted of 30 children (M/F 17/13, the median age was 14.0 years, the age range was from 12 to 17). These children did not have complaints of dyspeptic syndrome or abdominal pain. Children of the control group were not infected with HP. The urea breath test in these children gave negative results. These groups were homogeneous for sex and age and for socio-economic status as assessed by level of education and household size. All children included in the study did not take antibiotics during the last six months.

The study was approved by the Medical Ethics Review Committee. All children provided their written informed consent to participate in the study.

HP was carried out by two methods: the rapid urease test with biopsy using the test system "Helpil" ("AMA", Russia) and the urea breath test using the test system "Helic" with detector tubes ("AMA", Russia).

Diagnosis of SIBO was based on the non-invasive hydrogen breath test with a load of lactulose using the analyzer of exhaled hydrogen "LactofaH2" ("AMA", Russia). The test started with the measurement of the basal level of hydrogen. Then, the patients drank the test solution of lactulose. Level rise of $10 \mathrm{ppm}$ above baseline for 60 - $90 \mathrm{~min}$ was regarded as evidence of SIBO in a patient.

Statistical analysis of the obtained results was carried out in MedCalc Version 13.0.2.0. Qualitative variables are presented as percentages. To compare categorical variables between groups, the Chi-square test $\left(\chi^{2}\right)$ and the Fisher F exact test were used. A calculated difference of $\mathrm{p}<0.05$ was considered significant.

\section{Results}

SIBO was detected in $57(95.0 \pm 2.8 \%)$ school-age children with DUD, associated with HP, by the hydrogen breath test with a load of lactulose. In $37(74.0 \pm 6.2 \%)$ patients with functional dyspepsia, associated with HP, SIBO was determined. In the control group SIBO was diagnosed only in $4(13.3 \pm 6.2 \%)$ children (Figure 1$)$. The differences in frequency of SIBO between patients with DUD, children with functional dyspepsia, associated with HP, and children of the control group were statistically significant $[\mathrm{p}<0.05]$. 


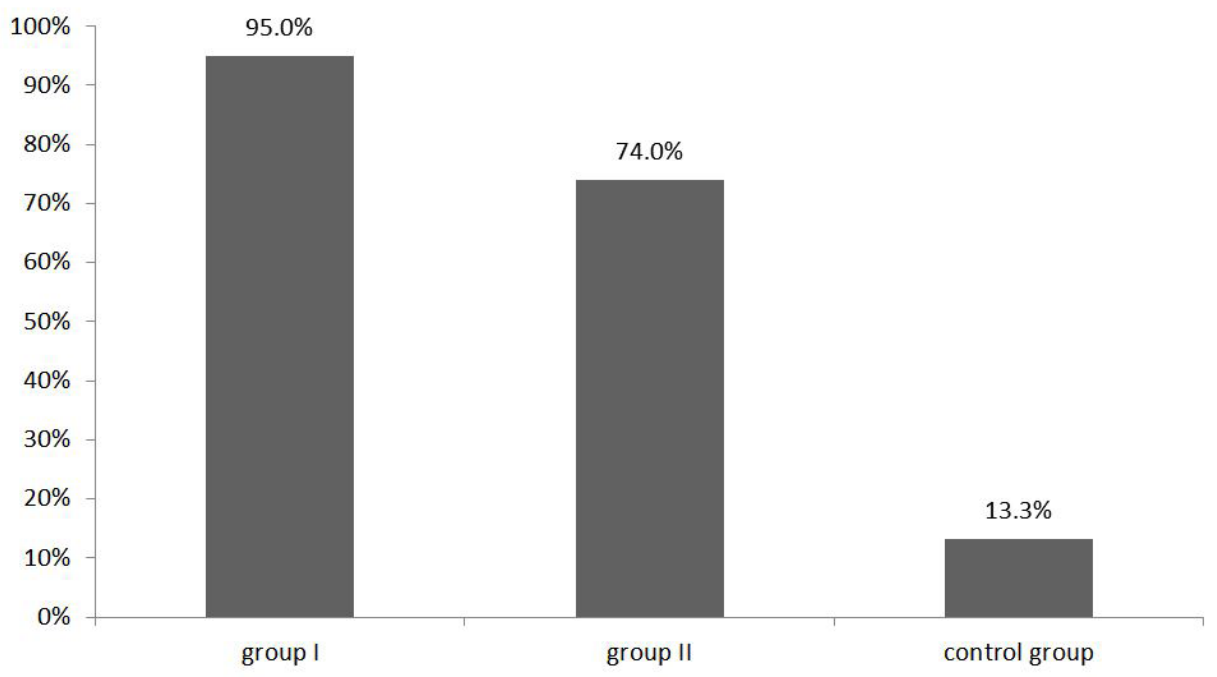

Figure 1: SIBO prevalence in patients surveyed groups

\section{Discussion}

In the study, we found that the using of the hydrogen breath test with a load of lactulose is fast, safe and effective method for diagnosing SIBO in school-age children. Tolerability of the test in all patients was good.

Analysis of the results showed that in children organic and functional gastroduodenal pathology in most cases is associated with increased intestinal microbial contamination of fecal microflora. It allows to the conclusion about the important role of SIBO in the pathogenesis of the pathological changes in the upper part of the gastrointestinal tract. We found that the progression of the inflammatory process and duodenal ulcer formation in children develops not only at the persistence of HP infection, but also on the background of SIBO.

Thus, an increase of microbial contamination of the small intestine by the hydrogen breath test with a load of lactulose was found in almost all children with DUD, associated with HP. It was found that functional dyspepsia, associated with HP infection, also occurs on the background of translocation fecal microflora in the small intestine. SIBO was diagnosed in $3 / 4$ patients with functional dyspepsia, associated with HP. Changes of gastric secretion and impaired motility of the gastrointestinal tract may play a starting role in the pathogenesis of not only functional dyspepsia but also SIBO. Violation of the microbial balance in the small intestine along with infection of HP in turn involved in further progression of chronic gastroduodenal pathology and the development of destructive inflammatory processes of the duodenal mucosa. These changes may be considered in the treatment of children with chronic gastroduodenal pathology, associated with HP infection.

\section{Conclusion}

This study demonstrated the high prevalence of SIBO in patients with DUD and functional dyspepsia, associated with HP. Microbiological disturbances in the intestine may play an important role in the pathogenesis of chronic gastroduodenal pathology, associated with HP in children.

\section{References}

1. Gold BD, Gilger MA, Czinn SJ (2014) New Diagnostic Strategies for Detection of Helicobacter pylori Infection in Pediatric Patients. Gastroenterol Hepatol (N Y) 10: 1-19.

2. Khalifa MM, Sharaf RR, Aziz RK (2010) Helicobactier pylori: a poor man’s gut pathogen? Gut Pathog 2: 2.

3. Bures J, Cyrany J, Kohoutova D, Forstl M, Rejchrt S, et al. (2010) Small intestinal bacterial overgrowth syndrome. World J Gastroenterol 16: $2978-90$.

4. Siniewicz-Luzeńczyk K, Bik-Gawin A, Zeman K, Bąk-Romaniszyn L (2015) Small intestinal bacterial overgrowth syndrome in children. Prz Gastroenterol 10: 28-32.

5. Shah SC, Day LW, Somsouk M, Sewell JL (2013) Meta-analysis: antibiotic therapy for small intestinal bacterial overgrowth. Aliment Pharmacol Ther 38: 925-34. 6. Grace E, Shaw C, Whelan K, Andreyev HJ (2013) Review article: small intestinal bacterial overgrowth- prevalence, clinical features, current and developing diagnostic tests, and treatment. Aliment Pharmacol Ther 38: 674-88.

7. Rezaie A, Pimentel M, Rao SS (2016) How to Test and Treat Small Intestinal Bacterial Overgrowth: an Evidence-Based Approach. Curr Gastroenterol Rep 18: 8. 8. Pimentel M (2016) Breath Testing for Small Intestinal Bacterial Overgrowth: Should We Bother? Am J Gastroenterol 11: 307-8.

9. Crane RJ, Jones KD, Berkley JA (2015) Environmental enteric dysfunction: an overview. Food Nutr Bull 36: S76-87. 
